\title{
Pre-Breakdown Arcing as Proxy for DC Dielectric Breakdown Testing of Polymeric Insulators
}

\author{
Allen Andersen and JR Dennison \\ Materials Physics Group \\ Utah State University \\ 4415 Old Main Hill, Logan, UT 84322 USA \\ allen.andersen@aggiemail.usu.edu,jr.dennison@usu.edu
}

\begin{abstract}
Pre-breakdown arcing is proposed as a key indicator of DC breakdown properties of polymeric dielectric materials. Voltage step-up tests were performed for films of lowdensity polyethylene (LDPE) at ramp rates significantly lower than is common in most studies, allowing for the observation of both breakdown and transient pre-breakdown current spikes. The distributions of the breakdowns versus applied field were compared with the more frequent pre-breakdown arcs. A strong correlation was observed for LDPE between the distribution of breakdowns in step-up tests and the distribution of prebreakdown arcing. Pre-breakdown arcing distributions are much easier to obtain than breakdown distributions and may be an efficient indicator of the minimum field at which breakdown can occur, leading to accelerated test methods. The possible physical origins underlying pre-arcing and its relation to breakdown distributions are discussed.
\end{abstract}

Keywords-arcing; breakdown; electrostatic discharge; polymers

\section{INTRODUCTION}

Standard step-up to breakdown tests are performed in order to determine the dielectric breakdown field strength of materials [1]. These tests performed on thin films samples can overestimate breakdown field strengths, especially when using rapid ramp rates recommended by standard procedures [2]. These destructive tests measure a distribution of breakdown fields; therefore, many tests performed on many samples are required to obtain a statistically significantly distribution of breakdowns. Accelerated test methods are advantageous, since it is impractical to test multiple candidate materials for the duration of the application lifetime which can be as long as many decades. Accelerated DC testing of dielectric materials would expedite the characterization and selection of insulating materials for HVDC, high voltage switching, spacecraft charging, electronics, and other applications [3].

Step-up to breakdown tests were performed on samples of low density polyethylene (LDPE). The breakdown events were observed together with transient, non-shorting current spikes that have been termed pre-arcing [4]. For each permanent and irreparable shorting breakdown event, many pre-arc events were observed as the applied electric field was ramped to breakdown. The distribution of the pre-arcs versus applied field is shown to match the form of the distribution of breakdown events which is commonly interpreted as the probability of failure at a given applied field [5].

We propose the distribution of pre-arcing events could be used as an efficient proxy for the distribution of electrostatic discharge (ESD) breakdown events commonly used to determine a material's dielectric strength. Since pre-arcing data can be obtained more rapidly than breakdown data sets of the same size, it is proposed that the test time necessary to acquire statistically significant data could be dramatically reduced.

\section{EXPERIMENTATION}

\section{A. Experimental Methods and Typical Results}

Samples of LDPE from Goodfellow $(29.7 \pm 2 \% \mu \mathrm{m}$ thick, density of $0.92 \mathrm{~g} / \mathrm{cm}^{3}$ [6], estimated crystallinity of $50 \%$ [7], and a peak fractional mass distribution of $\sim 6 \cdot 10^{3}$ amu $[8,9]$ ) were broken down in a parallel plate geometry under vacuum using a modified [2] ASTM method [10,11]. All samples were cleaned, vacuum baked while in contact with a grounded surface to eliminate absorbed water and volatile contaminants and any residual stored charge, and stored in dry $\mathrm{N}_{2}$ gas prior to testing [8]. Voltage across the samples was increased stepwise at $\sim 20 \mathrm{~V}$ per $3.5 \mathrm{~s}$ until breakdown was eventually observed, as marked by a steady increase in leakage current (see Fig. 1). Details are provided in [2].

A single step-up test results in a current versus voltage trace which can, in general, have four regions (see Fig. 1). In the low current region at low voltages, the leakage current is negligible. As the voltage increases, non-shorting transient current spikes or pre-arcs (typically ranging $\sim 1$ to $\sim 20 \mu \mathrm{A}$ ) are observed in a pre-arcing region. The ammeter used has a response (integration) time of $\sim 0.5 \mathrm{~s}$. Fast oscilloscope measurements show pulses $<5 \mu$ s, indicating that larger pre-arcs seen by the ammeter are the integrated current over several smaller pulses, as shown in the inset of Fig. 1. At high voltage, the ESD breakdown region is characterized by a transition to a steadily increasing current (typically on the order of $\sim 40 \mu \mathrm{A}$, as set by Ohm's law for the current limiting resistors in the test circuit). In many cases there is an abrupt transition from intermittent prearcing to steady breakdown current. However, an irregular current region of non-negligible but sub-ohmic erratic current can be observed. One interpretation of this irregular current is that pre-arcing becomes more frequent as the applied voltage

Research was partially funded by a NASA Space Technology Research Fellowship. 
approaches breakdown, to the point at which one or more prearcs occurs during every ammeter measurement of integrated current.

\section{B. Comments on Pre-Arcing}

This pre-arcing phenomenon is not widely reported in the literature. We note a recent article where similar non-shorting small amplitude currents spikes are reported as a side note to the main results [12]. When our test system was first used, the prearcing events were dismissed as noise. Upon closer inspection, it became clear that these events are clearly above the system noise and that the pre-arcing behavior changes from material to material [4].

Perhaps the primary reasons other studies have not widely reported pre-arcing are simply that they were not looked for and that the pre-arcing current amplitudes were much smaller or faster than most events of interest for typical breakdown studies. A number features of our experimental methods have been identified that may contribute to why similar pre-arcing events are not always seen in other similar experiments [2]. Most importantly, these are:

(i) The use of slower ramp rates than most standard tests [1]. At $20 \mathrm{~V} / 3.5 \mathrm{~s}$ an average of 17 unadjusted pre-arcs are observed above the noise envelope in a typical step-up test below the threshold of the irregular current region. Assuming that pre-arcing frequencies do not vary significantly with ramp rate, the chance of seeing even one pre-arc in a more rapid step-up test with the recommended ramp rate of $500 \mathrm{~V} / \mathrm{s}$ is only $\sim 19 \%$.

(ii) Ammeters used were sensitive down to $100 \mathrm{nA}$. ASTM standards do not specify current sensitivity, but rather recommend detecting breakdown by "a sensing element such as a circuit breaker, a fuse, or a current-sensing circuit" or an observation of a physical rupture in the sample [10,11]. These methods are likely to miss fast, small, non-shorting pre-arcing events. Even commonly used ammeters with less sensitivity will detect few pre-arcs; in our tests an average of $<5$ arc above $2 \mu \mathrm{A}$ per test were observed below the irregular current region.

Other experimental techniques that may also contribute include:

(iii) Stepwise ramping with sharp edges in the applied voltage profile used in these tests, may trigger more pre-arcs than a continuous voltage ramp often used [13].

(iv) Large flat electrode areas, rather than sharp needles often used in ESD tests [14].

(v) Beveled electrode edges that minimize edge effects [5].

(vi) Polished electrodes to minimize the effects of protrusions $[15,16]$.

(vii) Spring-loaded sample clamping system to maintain a uniform $E$ field.

(viii) Samples that extend well beyond the electrode area.

(ix) High quality samples of uniform thickness were used to minimize impurities.

(x) Samples were baked prior to testing, to remove any absorbed water or other volatiles [15].

(xi) Tests were performed under high vacuum $\left(<10^{-3}\right.$ Pa base pressure).

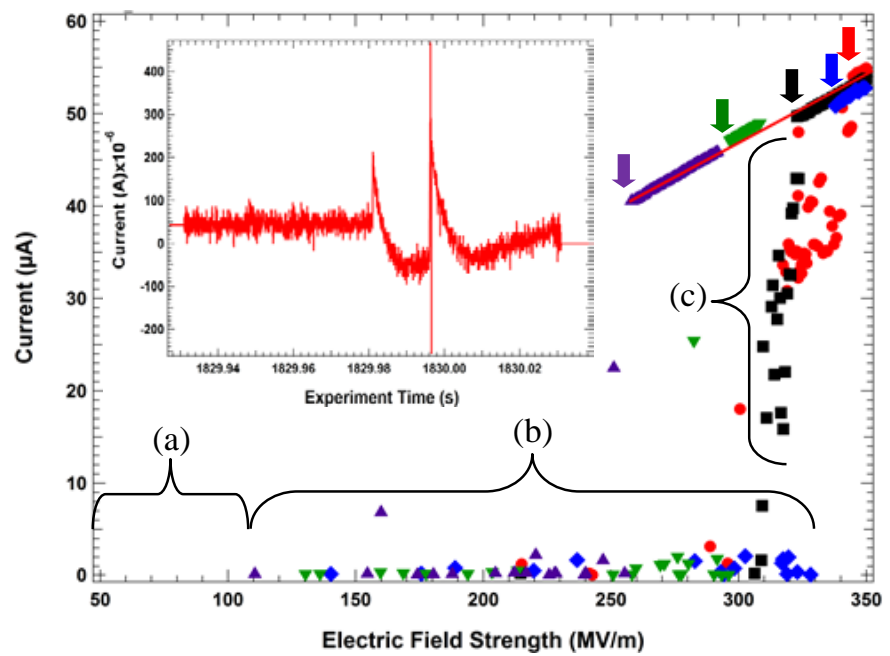

Fig. 1. Current traces from five step-up tests. (a) Low-current region-no arcing events are observed below $\sim 100 \mathrm{MV} / \mathrm{m}$. (b) Above this field, transient lowamplitude current spikes (pre-arcs) are observed. These pre-arcs tend to increase in magnitude and frequency until the sample eventually breaks down. Breakdowns, marked with colored arrows for each of the five tests, are characterized by a sudden transition to an ohmic slope corresponding to the current limiting resistors in the test circuit (red line). (c) Frequently the transition from intermittent pre-arcing to breakdown exhibits erratic sub-ohmic currents that do not return to negligible current before breakdown. The inset shows an oscilloscope trigger with multiple peaks corresponding to a single prearc event as measured by the ammeter [4]. Larger pre-arcs, as observed by the ammeter, are the result of current integrated over multiple smaller fast events.

As noted before, some pre-arcing events can be much larger than others. Several pre-arc events have been measured using a fast oscilloscope simultaneously with the ammeter (inset of Fig. 1) [4]. The arcs observed with the oscilloscope had widths of $\$ 5$ $\mu$ s (this may have been limited by the RC time constant of the measurements circuit) as compared to the ammeter's response time of $\sim 0.5 \mathrm{~s}$ This demonstrated that a single large amplitude pre-arc signature in the ammeter data can actually be multiple smaller fast pre-arcs integrated over the ammeter's data response time. As discussed below, large amplitude pre-arcs were interpreted as multiple small pre-arcs by dividing the prearc amplitude by a typical small arc value.

\section{StATISTICAL COMPARISON OF PRE-ARCING AND BREAKDOWNS}

The experiments presented here were originally intended to determine breakdown field strength. After many experiments, it was noted that the threshold voltage for pre-arcing appeared to be consistent with the minimum field for observed breakdowns [4]. Due to the high frequency of observed pre-arcing events, the pre-arcs could be used as an efficient indicator of breakdown behavior and the experiment time necessary to characterize a material could be reduced from many weeks to many hours. In fact, we present here evidence that the distribution of pre-arcing events over the full range of applied fields is very similar to the distribution of breakdown events in LDPE.

\section{A. Mathematical Methods}

Distributions of pre-arcing and breakdowns are compared quantitatively in this section. The empirical cumulative distribution (ECD) of observed events as a function of applied 


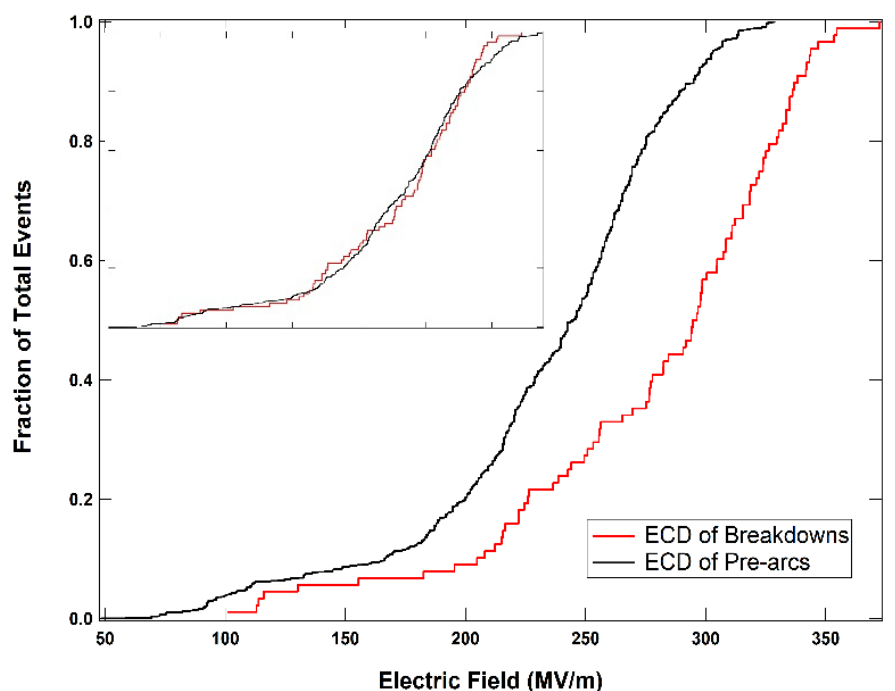

Fig. 2. Empirical cumulative distributions of pre-arcs and ESD breakdowns in LDPE. The reason the pre-arc curve falls well to the left of the breakdown curve is due to difficulty of counting pre-arcs at high fields as breakdown begins to occur in the irregular current region. A modified breakdown ECD addressing this discrepancy is compared with the pre-arcing ECD in the inset. Note that the pre-arc curve is smoother than the breakdown curve due to the higher data density.

field, $F$, for sufficiently large sample size $n$ of events $x_{i}$, is equivalent to a probability of occurrence and is given by

$$
\begin{array}{cc}
\hat{P}_{n}(F)=\frac{1}{n} \sum_{i=1}^{n} \mathbf{1}\left\{x_{i} \leq F\right\} \\
\text { where } \quad \mathbf{1}\left\{x_{i} \leq F\right\}=\left\{\begin{array}{lc}
1 & \text { if } x_{i} \leq F \\
0 & \text { otherwise }
\end{array} .\right.
\end{array}
$$

For breakdowns this calculation is straightforward in that each experiment yields one breakdown at a given field. Counting pre-arcs is more difficult because they are observed at many amplitudes. In order to more accurately count pre-arcs, a typical small pre-arc value $(0.15 \mu \mathrm{A})$ was estimated; larger amplitude arcs were divided by this small pre-arc value to determine nearest integer multiple of pre-arcs per ammeter measurement time. The shape of the resulting distribution of adjusted pre-arcs is quite insensitive to the choice of the typical small arc value, so long as this value is above the noise of the system (i.e., $\gtrsim 0.085 \mu \mathrm{A}$ ) and not so large as to eliminate too much data (i.e., $\lesssim 0.5 \mu \mathrm{A}$ ).

Furthermore, the counting is effected by what is considered a pre-arc. Due to the insensitivity of the resulting ECD and a relative abundance of pre-arcs, the pre-arcs were selected using a conservatively high threshold. An event was termed a pre-arc if there was a non-negligible current reading above our noise threshold and the current meter subsequently returned to negligible current at the next voltage step. In the high field irregular current region prior to breakdown (as observed in Fig. 1), there are often current readings below the ohmic breakdown line that do not return to negligible current. In this region near breakdown, it is often difficult to determine if there is a large quantity of frequent pre-arcing or if the sample is simply in the process of breaking down. Although many pre-arcs near breakdown fields were likely neglected, they were not included in the pre-arc ECD for the analysis of Fig. 2 due to the difficulty in counting them accurately. Using the methods described above 25,568 now quantized pre-arcs were compared to 88 ESD

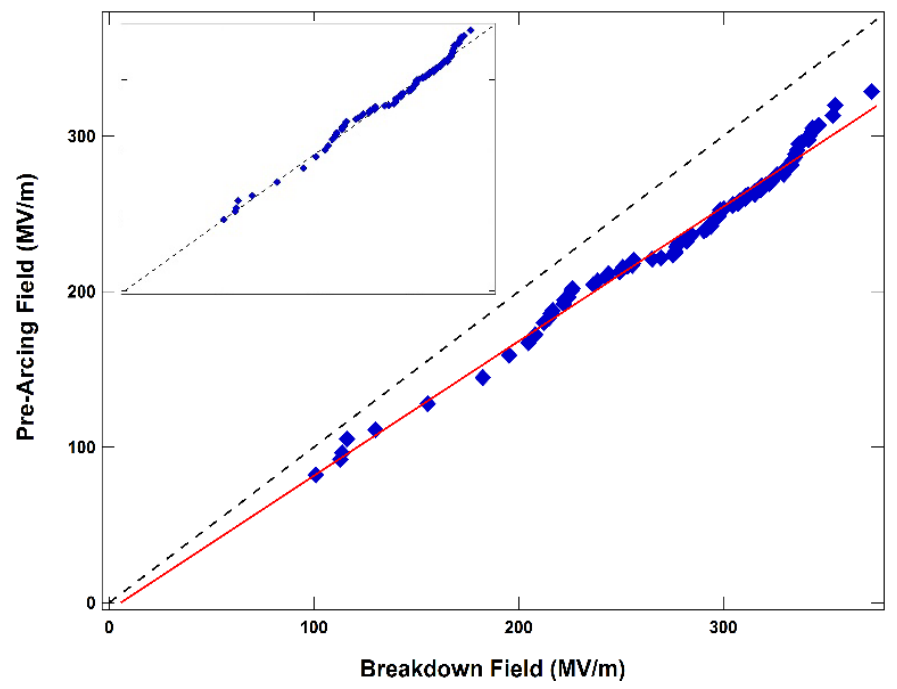

Fig. 3. Quantile-Quantile plot of pre-arcing and breakdowns. The red line is a liner fit to the data with a slope $0.86 \pm 0.01$, an intercept $-5 \pm 3$, and a correlation coefficient 0.993 . The inset shows the $\mathrm{Q}-\mathrm{Q}$ plot comparing the modified breakdown ECD to the pre-arc ECD. The shift away from unity slope is attributed to the difficulty in counting pre-arcs at fields close to breakdown. The lines $y=x$ (dashed black) with unity slope are shown for reference.

events. Although most of the pre-arc data are from the same set of 88 tests used for breakdown data, many are not. Again, the ECD of the pre-arcs is quite unresponsive to arbitrary changes how they are counted.

The ECD for the two distributions are shown in Fig. 2. Note that although the shapes of these distributions are quite similar, the pre-arcing curve lies to the left of the breakdown curve. This is an artifact of the difficulty in counting pre-arcing events as breakdown begins to occur. A modified pre-arcing ECD was calculated assuming pre-arcing over the whole range of ESD events, as is observed. The inset of Fig. 2 comparing the breakdown ECD to the modified pre-arc ECD, shows excellent overlap of the two distributions over the full range of applied fields.

Although Fig. 2 suggests strong similarities in the two distributions, a more advanced statistical technique is needed in order to make a quantitative comparison. The two distributions can be compared directly by constructing a quantile-quantile plot or Q-Q plot. The field values at which the likelihood (yaxis of Fig 2.), or quantile, of each event occurring is the same for the two distributions become the $\mathrm{x}$ and $\mathrm{y}$ values for the $\mathrm{Q}-\mathrm{Q}$ plot. Field values from the pre-arcing data set were linearly interpolated to match a quantile measured for each breakdown field value; a linear interpolation was deemed sufficient due to the much larger data density of the pre-arcing curve. Again, the inset shows the Q-Q plot comparing the modified breakdown ECD to the pre-arc ECD.

\section{B. Results and Interpretation}

The Q-Q plot of pre-arcing and breakdowns (Fig. 3) clearly shows that the distributions of these two phenomena are related. For two identical distributions, Q-Q points fall on a line with unity slope, zero intercept, and a linear correlation coefficient of unity. Q-Q points for two distributions with similar shape but a non-unity scaling factor-as is observed in Fig. 3-would be fit well with a linear model. The fit to data in Fig. 3 has a slope of 


\section{Proceedings of the 2015 IEEE Conference on Electrical Insulation and Dielectric Phenomena-(CEIDP)}

$0.86 \pm 0.01$ and an intercept of $-5 \pm 3$. The nearly unity linear correlation coefficient of 0.993 indicates an extremely significant correlation between the two ECD.

The non-unity slope is comparable to the left-ward shift of the pre-arcing distribution in Fig 2; as discussed before, this arises from the inaccuracy in counting pre-arcs in the irregular current region. The inset of Fig. 3 shows a Q-Q plot for the modified breakdown ECD for only fields up to the maximum field considered for pre-arcing events. This (slope 1.00 \pm 0.01 intercept $-0.1 \pm 4$, linear correlation coefficient 0.993) demonstrates that, for all fields between the threshold field and the lower boundary of the irregular current region, the modified breakdown and pre-arc ECD are very nearly identical.

\section{CONCLUSION}

This study has quantitatively shown that the distribution of pre-arcing events as a function of applied field is strongly correlated to the distribution of breakdown events in LDPE. Not only are the threshold fields for both ECD quite similar, but Q$Q$ plots show quantitatively that the shape of the two distributions over a wide range of fields have very similar shape. Having established that there is a correlation of pre-arcing with breakdown in LDPE, the next step is to test other materials to see if this relationship holds over a broader context. Testing and analysis for distributions of polyimide (Kapton HN), polypropylene, and borosilicate glass using the methods described here are currently in progress.

Although the analysis presented above is entirely empirical, we offer here a plausible physical context [2, 4, 17]. Consider low-energy physical defects such as bond bending. Defects with energies on the order of $k T$ have a finite probability of being thermally annealed [2], and are thus recoverable and selfhealing. Pre-arcing may result from a small avalanche of charge that, by releasing heat to its surroundings, thermally annihilates the defects necessary for further propagation thereby averting complete ESD breakdown. Alternatively, some discharges may continue until shorting breakdown occurs, especially if there is a sufficient density of high-energy chemical defects (e.g., broken bonds) that will not be thermally annealed [2]. In this way, we see that it is physically possible for both pre-arcing and ESD to have the same threshold field.

The relationship established between pre-arcing and breakdown could be a valuable tool in expediting the characterization of the breakdown threshold field for a given sample material, given the high frequency of pre-arcing events in a single voltage step-up test. Many pre-arcs are observed in the course of just a few step-up tests and the pre-arcing threshold value can be determined and used as proxy for the threshold field of ESD breakdown, thereby accelerating the characterization of a material's breakdown threshold. Further, the pre-arc distribution is very similar to the breakdown distribution over a full range of applied fields from this threshold field to a field where pre-arcing becomes difficult to measure. Compared to static voltage endurance time tests which individually last days to weeks or the prospect of taking many step-up tests, the analysis of pre-arcing has the potential to characterize a material in a matter of hours $[2,4]$.

\section{ACKNOWLEDGMENT}

We gratefully acknowledge insightful discussions with John Stevens and Dave Brown, as well as help with instrumentation and experiments from current and previous members of the Utah State University Materials Physics Group including Charles Sim, Dan Arnfield and Anthony Thomas.

\section{REFERENCES}

[1] ASTM, "Standard Specification for Polymeric Resin Film for Electrical Insulation and Dielectric Applications." vol. D5213-12, 2012.

[2] A. Andersen, J. R. Dennison, A. M. Sim, and C. Sim, "Electrostatic Discharge and Endurance Time Measurements of Spacecraft Materials: A Defect-Driven Dynamic Model," Plasma Science, IEEE Transactions on, 2015, in press.

[3] P. Trnka, M. Sirucek, M. Svoboda, and J. Soucek, "Condition-based maintenance of high-voltage machines-a practical application to electrical insulation," Electrical Insulation Magazine, IEEE, vol. 30, pp. 32-38, 2014

[4] A. Andersen and J. Dennison, "Pre-breakdown Arcing and Electrostatic Discharge in Dielectrics under High DC Electric Field Stress," 2014 Annual Report Conference on Electrical Insulation and Dielectric Phenomena, pp. 63-66, 2014.

[5] C. Chauvet and C. Laurent, "Weibull statistics in short-term dielectric breakdown of thin polyethylene films," Electrical Insulation, IEEE Transactions on, vol. 28, pp. 18-29, 1993.

[6] "Material Information-Polyethylene Low Density LDPE," Goodfellow, Ed. Devon, PA, 2006.

[7] H. J. Wintle, "Conduction Processes in Polymers," in Engineering Dielectrics-Volume IIA: Electrical Properties of Solid Insulating Materials: Molecular Structure and Electrical Behavior. vol. IIA, R. Bartnikas, Ed. Philadelphia, PA: Am. Soc. Testing and Materials, 1983.

[8] J. Brunson, "Measurement of charge decay time and resistivity of spacecraft insulators using charge storage method and application to theoretical modeling of charging behavior of insulators," Physics. PhD, Utah State University, Logan, UT, 2009.

[9] A. Peacock, Handbook of polyethylene: structures: properties, and applications: CRC Press, 2000.

[10] ASTM, "Standard Test Method for Dielectric Breakdown Voltage and Dielectric Strength of Solid Electrical Insulating Materials Under DirectVoltage Stress." vol. D3755-14, 2014.

[11] ASTM, "Standard Test Method for Electric Breakdown Voltage and Dielectric Strength of Solid Electrical Insulating Materials at Commercial Power Frequencies." vol. D149-97a, 2004.

[12] I. Rytöluoto, K. Lahti, M. Karttunen, and M. Koponen, "Large-area dielectric breakdown performance of polymer films-Part I: measurement method evaluation and statistical considerations on areadependence," Dielectrics and Electrical Insulation, IEEE Transactions on, vol. 22, pp. 689-700, 2015

[13] H. Wang, P. Zongren, L. Naiyi, Z. Shiling, G. Zihao, and Z. SiYu, "Transient electric field calculation of UHV GIS spacer under lightning impulse," in Electrical Insulation and Dielectric Phenomena (CEIDP), 2014 IEEE Conference on, 2014, pp. 542-545.

[14] L. A. Dissado and J. C. Fothergill, Electrical Degradation and Breakdown in Polymers. London, UK: The Institution of Engineering and Technology, 1992.

[15] L. Zavattoni, R. Hanna, O. Lesaint, and O. Gallot-Lavallee, "Dark current measurements in pressurized $\mathrm{SF}_{6}$ : Influence of relative humidity and temperature," in Electrical Insulation and Dielectric Phenomena (CEIDP), 2014 IEEE Conference on, 2014, pp. 23-26.

[16] L. Arevalo and W. Dong, "Effect of high dielectric protrusions on the breakdown phenomena of large electrodes under positive switching impulses," in Electrical Insulation and Dielectric Phenomena (CEIDP), 2014 IEEE Conference on, 2014, pp. 51-54.

[17] A. Andersen and J. R. Dennison, "Mixed Weibull Distribution Model of DC Dielectric Breakdowns with Dual Defect Modes," submitted to Proceedings of the 2015 IEEE Conference on Electrical Insulation and Dielectric Phenomena-(CEIDP 2015), 20. 